\title{
Influência dos currículos (formal e oculto) na formação da identidade profissional dos estudantes de medicina
}

\author{
Influence of (formal and hidden) syllabus in the \\ formation of medical students' professional \\ identity
}

\section{Jacqueline Rego Tardin ${ }^{1}$ lêda Aleluia² (1)}

\begin{abstract}
${ }^{1}$ Autora para correspondência. Escola Bahiana de Medicina e Saúde Pública (Salvador). Bahia, Brasil. jacquelinetardin14.1@bahiana.edu.br
\end{abstract} ²Escola Bahiana de Medicina e Saúde Pública (Salvador). Bahia, Brasil. iedaleluia@bahiana.edu.br

RESUMO | INTRODUÇÃO: A identidade profissional do estudante de medicina é formada durante a graduação e está sujeita à interferência de fatores inclusos no currículo formal e currículo informal do estudante. OBJETIVOS: Descrever as influências que o currículo formal e o currículo oculto exercem na formação da identidade profissional dos estudantes do curso de medicina da Escola Bahiana de Medicina e Saúde Pública. MÉTODos: Trata-se de um estudo transversal, descritivo, com metodologia qualitativa-quantitativa. Foi aplicado um questionário para avaliação dos estudantes sobre atividades práticas e teóricas ocorridas durante a graduação, inserção do humanismo na prática acadêmica além da descrição sócio demográfica e do perfil do grupo, sendo essas perguntas enviadas a 200 participantes. Os dados quantitativos foram analisados com o programa estatístico SPSS 20.0, considerando como estatisticamente significante $p<0,05$ e os dados qualitativos pela análise e categorização de conteúdo. RESULTADOS: 111/200 estudantes responderam o questionário, sendo a maioria proveniente do $8^{\circ}$ semestre da instituição. Houve percepção da influência de mentores, da contribuição do currículo formal e informal e da socialização na formação da identidade profissional do estudante. A análise das falas dos participantes mostrou a internalização da humanização se dá principalmente através a importância do exemplo, o contato com o paciente e a importância da formação prévia de caráter e personalidade do estudante. CONCLUSÃO: Conclui-se que há influência da matriz curricular e do corpo docente na construção da identidade profissional do estudante de medicina, além da evidência da necessidade de novas pesquisas que descrevam o impacto do currículo oculto.

PALAVRAS-CHAVE: Educação médica. Currículo. Currículo oculto. Identidade profissional.

\begin{abstract}
BACKGROUND: The professional identity of the medical student is formed during graduation and is subject to the interference of factors included in the formal curriculum and informal curriculum of the student. PURPOSE: This study aims to describe the influences that the formal curriculum and the hidden curriculum exert in the formation of the professional identity in the students of the medical course. METHODS: This is a cross-sectional, descriptive study with qualitative-quantitative methodology. A questionnaire was applied for the evaluation of the students about activities that occurred during graduation, insertion of humanism in academic practice, and socio-demographic description and group profile, and 200 participants received these questions. Quantitative data were analyzed using the statistical program SPSS 20.0, considering as statistically significant $p<0.05$ and qualitative data by content analysis and categorization. RESULTS: $11 / 200$ students answered the questionnaire, the majority coming from the 8th semester of the institution. There was perception of the influence of mentors, the contribution of the curriculum and socialization in the formation of the student's professional identity. The analysis of the participants' speeches showed the internalization of the humanization occurs mainly through the importance of example, the contact with the patient and the importance of the previous formation of character and personality of the student. CONCLUSIONS: There is influence of the graduation and faculty in the professional identity of the medical student, in addition to the evidence of the need for new research that describes the impact of the hidden curriculum.
\end{abstract}

KEYWORDS: Medical education. Syllabus. Hidden syllabus. Professional identity. 


\section{Introdução}

A identidade dos estudantes não é fixa, estando em constante processo de mudança. Para exercer medicina, assim como outra profissão, os estudantes precisam desenvolver uma identidade profissional e, a maneira pela qual a desenvolvam, tem implicações em seu futuro acadêmico e pessoal ${ }^{1}$. Desse modo, a formação da identidade profissional desempenha uma importância crucial na transição do papel do estudante de medicina para o de médico²

Pode-se definir identidade profissional médica como "uma representação de si mesmo" alcançada por etapas ao longo do curso durante o qual as características, valores e normas da profissão médica são internalizadas resultando em um modo de pensar, agir e se sentir como um médico² . Ela se fundamenta em domínios como comportamento humanizado, características pessoais de liderança, resiliência e empatia ${ }^{3}$.

Os alunos aprendem a se tornar médicos nos ambientes clínicos e acadêmicos ${ }^{4}$. As instituições estabelecem modelos de comportamento que são incorporados pelos estudantes. Nesse interim, além do ensino formal exercido pelas universidades, o estudante também aprende pela observação, e interação, de comportamentos dos colegas, dos médicos docentes e não docentes ao longo de sua formação .

Os ambientes de aprendizagem que afetam a formação da identidade profissional são vários e incluem salas de aula, hospitais e ambulatórios e áreas informais onde a socialização ocorre ${ }^{5,8}$. Ao contrário do currículo formal que ocorre na sala de aula através de aulas e seminários, o currículo oculto exemplifica o "processo cultural" de formação médica através da socialização dos médicoṣ. O currículo oculto exerce grande influência sobre a identidade profissional do estudante. Ele se refere ao conjunto de práticas que não são ensinadas explicitamente numa instituição, mas que são vivenciadas e internalizadas pelos estudantes e podem influenciar valores, atitudes e comportamentos. São impactos não intencionais e podem trazer contribuições positivas ou negativas para o futuro profissional dos alunos ${ }^{10}$.

Nesse contexto, práticas como a cerimônia do Jaleco Branco, responsável por conferir simbolicamente a transição para a profissão médica, o juramento de Hipócrates, atividades em laboratório de anatomia, sessões de aprendizagem baseado em problemas, seminários de ensino, inserção dos alunos em ambiente cirúrgico e adoção de uma linguagem particular com vocabulário específico, são importantes na graduação e marcam transições na identidade profissional do estudante $\mathrm{e}^{1,4,5}$.

O objetivo principal desse estudo é descrever as influências que o currículo formal e o currículo oculto exercem na formação da identidade profissional dos estudantes do curso de medicina da Escola Bahiana de Medicina e Saúde Pública. E, dessa forma, verificar, se no decorrer do curso, as práticas exercidas na graduação influenciam a construção de identidade do estudante. Além disso, entender como o currículo e o corpo docente podem influenciar na formação da identidade profissional do estudante de medicina e identificar a inserção de temas de humanismo no currículo e sua influência na formação do estudante.

\section{Métodos}

Trata-se de um estudo de corte transversal, de natureza observacional com abordagem quantitativa e qualitativa envolvendo alunos cursando os $8^{\circ}$ e $9^{\circ}$ semestres do curso de Medicina da Escola Bahiana de Medicina e Saúde Pública durante o período de 2017.1. A população do estudo consistiu em convidar os 200 alunos cursando os semestres citados acima. Trata-se de uma amostra de conveniência, parte da população estudada estava terminando o ciclo inicial de 04 anos e a outra parte estava entrando no internato.

Foram incluídos na pesquisa somente os estudantes de medicina, maiores de 18 anos, cursando os referidos semestres e que concordaram com o TCLE.

O estudo foi realizado através da aplicação de um questionário projetado pelas pesquisadoras, com base na literatura e na observação das práticas dos estudantes, contendo questões (fechadas e abertas) relacionadas às práticas formais e informais exercidas durante a graduação, e sobre o currículo da Escola Bahiana de Medicina e Saúde Pública, bem como perguntas referentes à formação e informações sociodemográficas dos estudantes. Buscou-se, assim, conhecer práticas referentes à graduação em medicina que possam de alguma forma influenciar a formação da identidade profissional do estudante de medicina. 
O projeto foi aprovado pelo Comitê de Ética em Pesquisa (CEP) da Escola Bahiana de Medicina e Saúde Pública, sob o número do CAAE 685603 17.9.0000.5544. Foi redigido um Termo de Consentimento Livre e Esclarecido para todos os participantes, a fim de esclarecer a relevância do projeto.

A análise estatística foi descritiva, realizada pelo programa IBM SPSS Statistics20.0. As variáveis numéricas foram analisadas seguindo as tendências central e de dispersão das variáveis conforme apropriado (variável contínua ou categórica) para a caracterização da casuística e apresentação dos resultados.

A análise qualitativa, das respostas às questões abertas, foi realizada através da metodologia de análise do conteúdo segundo Bardin, na modalidade temático-categorial ${ }^{6,7}$.

\section{Resultados}

\section{Dados quantitativos}

Participaram do estudo 111 indivíduos e todos responderam ao questionário. A média de idade dos estudantes foi de 23,1 anos (DP: +/- 2,3). Dentre estes, 74 (66,7\%) estudantes eram do sexo feminino. Considerando-se os semestres incluídos na pesquisa, $75(67,6 \%)$ dos indivíduos estavam no $8^{\circ}$ semestre e $36(32,4 \%)$ no $9^{\circ}$ semestre de graduação (Tabela 1).

Tabela 1. Características demográficas dos estudantes incluídos no estudo

\begin{tabular}{|c|c|}
\hline \multicolumn{2}{|l|}{ Característica } \\
\hline Idade (anos) & Média/DP \\
\hline \multicolumn{2}{|l|}{ Mínima: 20} \\
\hline \multicolumn{2}{|l|}{ Máxima: 32} \\
\hline & $23,1(+/-2,3)$ \\
\hline Sexo & n (\%) \\
\hline Feminino & $74(66,7)$ \\
\hline Masculino & $37(33,3)$ \\
\hline Semestre & n (\%) \\
\hline $8^{\circ}$ & $75(67,6)$ \\
\hline $9^{\circ}$ & $36(32,4)$ \\
\hline Estado Civil & n (\%) \\
\hline Solteiro(a) & $100(99,1 \%)$ \\
\hline União Estável & $1(0,9 \%)$ \\
\hline
\end{tabular}

Fonte: Banco de dados da pesquisa. 
Em relação a religião observou-se que $49(44,1 \%)$ eram católicos, seguidos pelos espiritas $21(18,9 \%)$ e $23(20,7 \%)$ se definiram como sem religião. Quando analisada a formação profissional prévia, 106 (95,5\%), dos participantes, não possuíam graduação anterior e $5(4,5 \%)$ já haviam cursado outra graduação antes do curso de Medicina. Dentre os que já cursaram algum curso, 4 (80\%) tinham graduação em outra área de saúde. Apenas um participante (20\%) possuía graduação numa área de exatas.

Em relação à atividade extracurricular, 100\% dos estudantes informaram já ter participado de alguma atividade em determinado momento do curso. As mais frequentes por ordem decrescente foram participação em Liga Acadêmica, Monitoria e Estágio (Gráfico 1).

Gráfico 1. Frequência de participação em atividades extracurriculares dos estudantes participantes no estudo

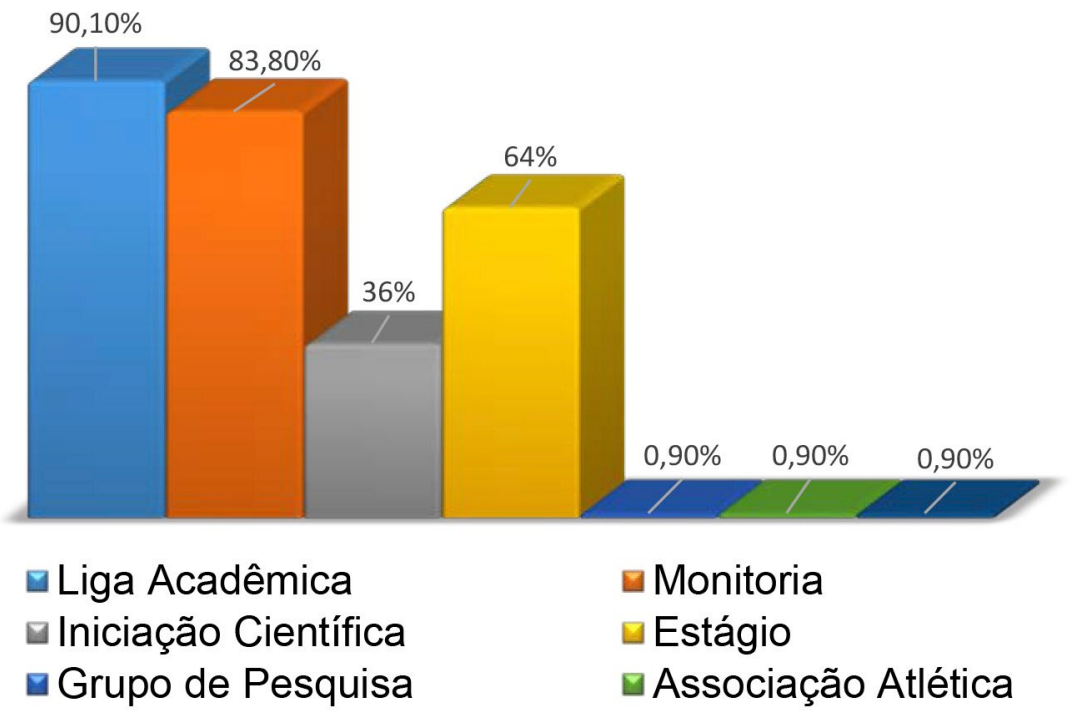

Quando perguntados se já haviam estudado no exterior, foi observado que $89(80,2 \%)$ não realizaram nenhuma das modalidades disponibilizadas para estudo no exterior. Dos estudantes que declararam já ter estudado fora do país de origem, 21 (18,9\%) participantes fizeram a partir de programa de intercâmbio, $1(0,9 \%)$ através do programa do governo federal Ciências sem Fronteiras e $1(0,9 \%)$ durante viagem realizada a lazer.

Questionando-se a humanização durante as aulas práticas no laboratório de anatomia, 36,9\% dos estudantes acreditam fortemente que é realizada de forma adequada. Em relação à realização do ritual da oração ao cadáver, 36\% concordam fortemente que a prática contribui pra humanização que a instituição de ensino adota. (Gráfico 2). 
Gráfico 2. Opinião dos participantes acerca da humanização a partir de práticas realizadas na instituição de ensino

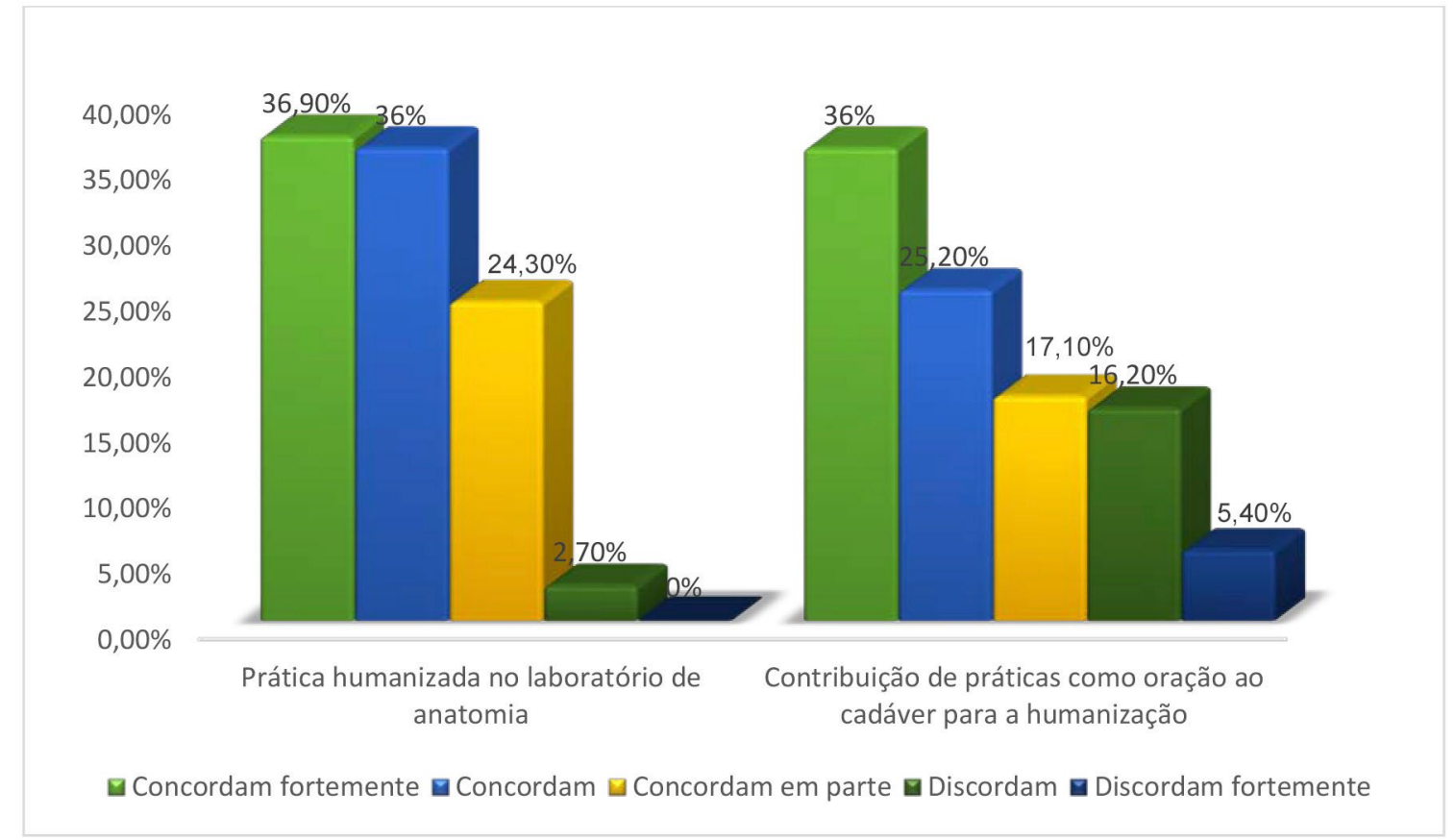

Uma vez perguntado se o contato com o paciente em âmbito ambulatorial ajudaria a estabelecer uma relação médico-paciente humanizada, $68,5 \%$ concordam fortemente, $25,2 \%$ concordam e nenhum participante discorda dessa prática.

Sobre o currículo formal de medicina, incluindo ambulatório e atividades cirúrgicas, 85,6\% dos participantes acreditam que as práticas abordadas são pautadas no humanismo e na relação médico-paciente. Quando inquiridos se o uso do método Problem Based Learning (PBL) cumpre o propósito de estabelecer discussões clínicas centradas no paciente, $74(66,7 \%)$ dos estudantes acreditam que o método cumpre esse papel, enquanto que $37(33,3 \%)$ discordam desse objetivo.
Analisando se algum professor/médico/mentor já havia adotado alguma conduta considerada positiva ou negativa na relação médico-paciente, $97,3 \%$ dos estudantes concordam que a postura do mentor é geradora de influência nesse aspecto. Todos os estudantes afirmam já ter presenciado alguma situação com professor/médico/mentor que poderia gerar uma influência positiva ou negativa sobre os mesmos.

Do total de participantes do estudo, 85,6\% afirmam já ter se dedicado mais a alguma matéria por causa de um professor. Levando-se em consideração a influência que um professor/médico/mentor poderia exercer sobre a escolha de uma especialidade, a maioria $(47,9 \%)$ concorda fortemente que haja interferência do profissional na escolha da especialidade por parte do estudante (Gráfico 3). 


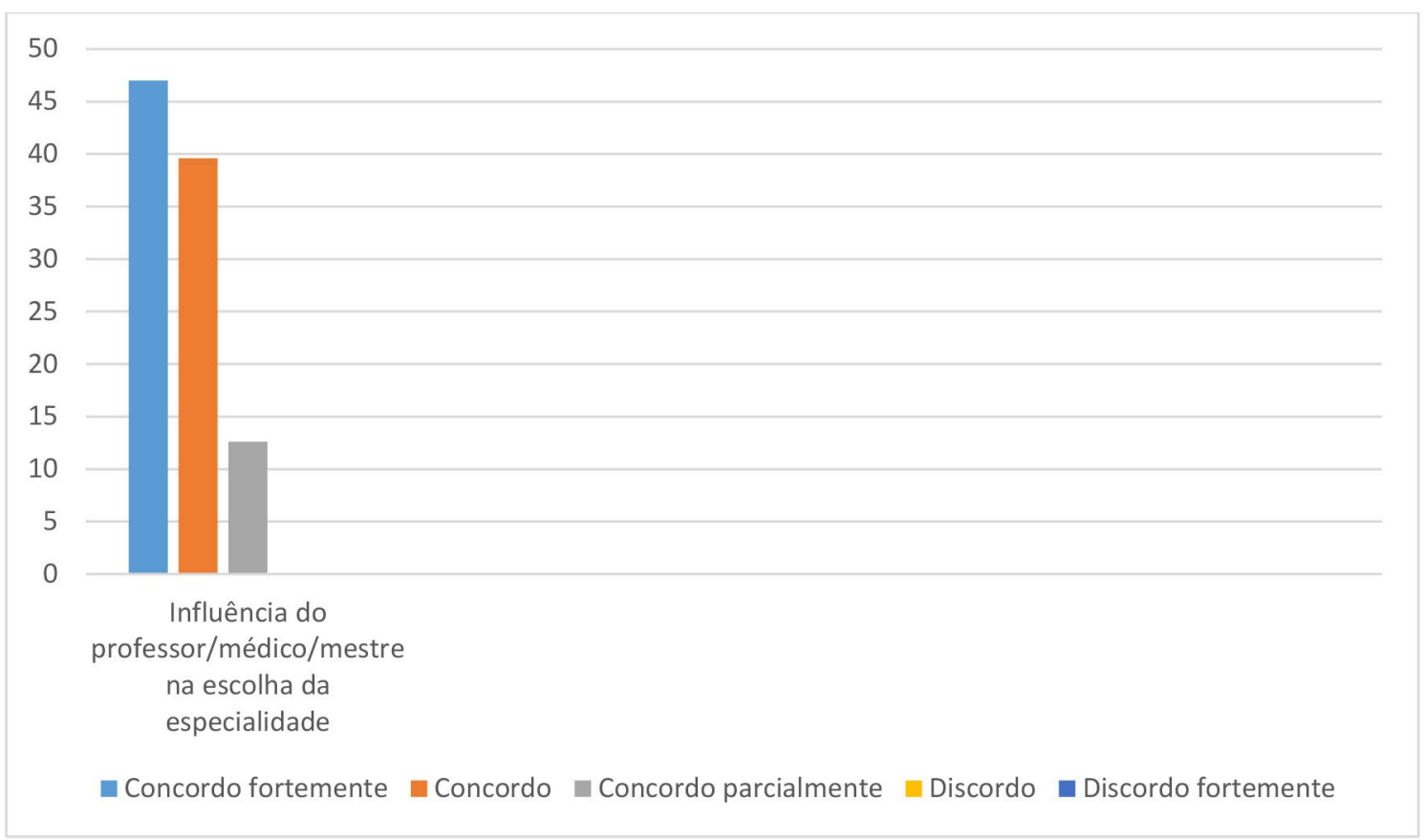

Uma vez questionados se a faculdade influencia o comportamento e o padrão de vestimenta dos alunos, $29,4 \%$ concordam fortemente e $36,9 \%$ concordam que o meio acadêmico interfere no seu modo de vestir. Apenas $7,2 \%$ dos participantes discordam que a faculdade contribua para esse aspecto.

\section{Dados qualitativos}

Quando perguntados se a humanização da medicina praticada em situações informais é mais internalizada do que quando ensinada durante as aulas, a percepção dos estudantes é que essa prática é mais incorporada quando praticada informalmente.

Dos que concordaram, surgem três categorias que são: a importância do exemplo, o contato com o paciente e importância da formação prévia de caráter e personalidade do estudante.

Em relação à sensibilização e inspiração que o contato com o professor sugere através de exemplos, foram extraídos o seguintes fragmentos:

"Sim. A prática do que é ensinado é mais importante e impactante do que a teoria. Sensibiliza e inspira mais o próprio estudante".
"Diria que o exemplo é a melhor forma: Falar "precisamos ser humanos" ou similar durante as aulas não nos marca. O que fica para o estudante é aquele paciente que traz um problema "psicológico" maior do que o problema "biológico" apresentado nas queixas. Por várias vezes que um paciente desses me apareceu, nem sequer soube perceber esse problema ou quando consegui, não soube lidar. Nas situações que isso aconteceu e tive o prazer de ter um médico-professor atencioso, que tanto soube colher a real história do paciente em minha frente quanto discutir comigo o que eu senti durante aquela conversa, com certeza houve um aprendizado muito grande."

Já referente à vivência com o paciente, foi extraído o fragmento abaixo:

"A humanização na prática informal é muito mais internalizada, pois garante o acesso à vivência da rotina médica e portanto, a proximidade com o paciente"

Em relação ao fato de que a humanização se referia à própria formação do ser, foi evidenciada a seguinte fala:

"Sim! A Humanização na medicina só é praticada com a humanização de nós mesmos, e, por mais que a faculdade ajude bastante, isso é algo que só a vida pode nos ensinar". 
Os participantes que concordaram em parte com humanização em ambientes informais, sugeriram que o aspecto formal nesse caso também é relevante.

"Em parte. Acredito que a humanização da medicina é mais factível e possível de ser internalizada no momento de discussão de casos e do "presenciamento" de atitudes holísticas no momento das orientações dadas diretamente ao paciente. Ou seja, presenciar atitudes de humanização no discurso do médico com o paciente contribuem para a internalização de práticas mais humanizadas. Além disso, o treinamento prático em laboratórios ou situações de simulação podem contribuir tanto quanto para a internalização de tais práticas."

Diante do exposto acima, foi construído um esquema, na tentativa de estabelecer a relação entre as falas dos estudantes e percepção da pesquisadora sobre a internalização da humanização.

Figura 1.

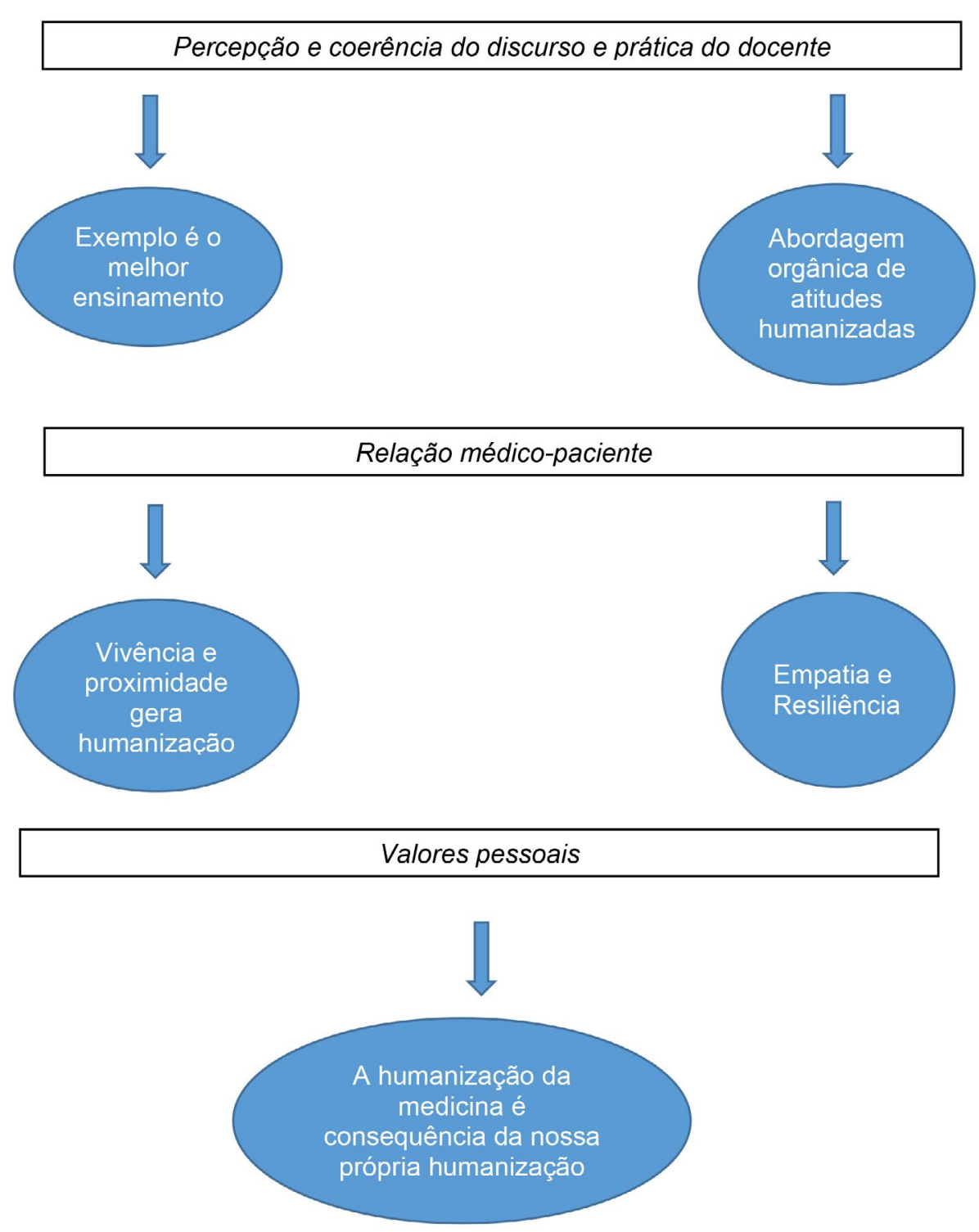


Esse estudo buscou avaliar a influência que a graduação (currículo formal e informal) exerce na formação da identidade profissional. Foi realizada uma análise da influência do currículo oculto em práticas exercidas na graduação, bem como atividades que compõem o currículo formal, observando a prática de humanização, entre os alunos da Escola Bahiana de Medicina e Saúde Pública.

Foi observado que a transição de estudantes de medicina para médicos desempenha um importante papel na formação da identidade profissional do aluno ${ }^{2,5}$. A literatura contempla essa transformação a partir da visão do médico como educador e, em poucos estudos se tem a perspectiva do estudante da construção dessa identidade durante a vida acadêmica. Nesse contexto foi constatado que práticas como cerimônia do jaleco branco e oração ao cadáver implementadas em algumas instituições de ensino médico podem influenciar essa construção? Esses rituais constituem um processo de passagem do estudante recém-ingressado no meio acadêmico se estabelecendo como um dado relevante, onde os alunos aprendem o significado de responsabilidade e expectativas para o humanismo e profissionalismo ${ }^{11,18}$. São ritos de passagem que estão presentes ao longo do curso, e se tornam marcos de reflexão e construção identitária.

Quanto à análise do currículo formal, ao se deparar com práticas em laboratórios de anatomia e o ensino do $\mathrm{PBL}^{14}$ o estudante encontra uma visão humanizada dessas atividades. Há, então, a promoção de ética, princípios e habilidades de raciocínio. A prática de tais atividades contribui, portanto, para o despertar do olhar humano do agora acadêmico sobre os métodos de ensino e ferramentas utilizadas no processo de aprendizagem ${ }^{15}$, que terão repercussões sobre o comportamento do profissional futuro perante o seu paciente. Além disso, ajuda os alunos a despertarem uma compreensão rica e multifacetada da identidade profissional.

A análise do currículo oculto como um gerador de impactos(valores, atitudesecomportamentos)nãointencionais que podem assumir a forma de contribuições positivas e negativas ${ }^{14}$ sobre o estudante é confirmada nesse estudo. Nesse interim, a partir dos questionamentos sobre a influência do mentor, observando o papel e conduta do mesmo, grande parte dos estudantes referiram que há contribuições positivas e negativas. Diante disso há a relevância do aspecto de "role modeling"16 e a importância do processo cultural gerado através dos diferentes processos de socialização durante a faculdade, principalmente relacionado aos mentores/médicos. Experiências de aprendizado negativas podem afetar negativamente o desenvolvimento da identidade profissional do estudante de medicina e serem perpetuadas entre os estudantes através de seus círculos sociais. Entretanto, quando os valores, comportamentos e padrões éticos são positivos, tanto a identidade profissional como o profissionalismo ${ }^{17}$ a ser construído pelo estudante são beneficiados, como por exemplo com o desenvolvimento de atitudes humanísticas ${ }^{16,18}$. A socialização então, se torna importante quando há relatos otimistas sobre os cuidados com os paciente e valores profissionais.

Ainda sobre aspectos da relação docente-discente, foi evidenciado que o discurso do professor/médico/ mentor tem influência sobre a predileção do aluno por determinada disciplina, bem como a possibilidade de haver interferência direta ${ }^{5,19}$ sobre o comportamento do futuro profissional, além da escolha da especialidade médica pelo estudante.

Ao se questionar se atividades ambulatoriais e de clínicas cirúrgicas envolveram humanismo em sua prática foi observado que o contato inicial entre paciente e estudante se destaca como elemento essencial na formação da identidade profissional do estudante de medicina. Este dado corresponde à literatura na medida em que essas práticas fornecem uma aprendizagem complexa para o estudante ao adotar uma abordagem holística, pautada em temas como a reflexão do relacionamento médico-paciente e a construção de empatia e resiliência ao longo desse contato $10,11,20$. Esse destaque é visto nos discursos dos estudantes.

Quando analisado se a faculdade influencia o comportamento e padrão de vestimenta dos alunos, foi observado que há uma evolução gradual para vestir-se e portar-se como médico e que, dessa forma, 
o currículo oculto pode ser evidenciado no vestuário dos estudantes 14,19 , bem como representa uma transição na identidade profissional do estudante.

\section{Considerações finais}

Uma vez que a identidade profissional médica é a representação de si mesmo no decorrer do curso de medicina alcançada quando características, valores e normas da profissão médica são incorporadas e resultam em um pensamento individual de agir e se sentir médico ("think, act and feel like a physician") 3,4.

A maioria dos estudos considera que o currículo oculto traz mais contribuições negativas do que positiva durante a graduação do estudante ${ }^{13,18,21}$, visto que ainda é muito difícil identificar todos os fatores inclusos nesse currículo. O nosso estudo mostrou que, apesar de existirem práticas formais no currículo, previstas no plano de ensino das disciplinas curriculares, os estudantes internalizam mais a humanização da medicina por meio de observação de exemplos e atitudes de mentores e colegas, além do que o contato com o paciente traz uma nova percepção de empatia e resiliência necessária durante a jornada acadêmica. E dessa forma o futuro profissional vai se formando gradativamente, trazendo à tona características de identidade social médica.

\section{Contribuições das autoras}

Tardin JR desenhou o projeto, coletou e analisou os dados e escreveu o artigo. Aleluia I analisou os dados, e supervisionou a pesquisa, escrita e revisão.

\section{Conflitos de interesses}

Nenhum conflito financeiro, legal ou político envolvendo terceiros (governo, empresas e fundações privadas, etc.) foi declarado para nenhum aspecto do trabalho submetido (incluindo, mas não se limitando a subvenções e financiamentos, participação em conselho consultivo, desenho de estudo, preparação de manuscrito, análise estatística, etc.).

\section{Referências}

1. Goldie J. The formation of professional identity in medical students: Considerations for educators. Med Teach. 2012;34(9):e641-8. doi: 10.3109/0142159X.2012.687476

2. Cruess RL, Cruess SR, Boudreau JD, Snell L, Steinert Y. Reframing medical education to support the development of a professional identity. Acad Med. 2014;89(11):1446-51. doi: 10.1097/ACM.0000000000000427

3. Holden MD, Buck E, Luk J, Ambriz F, Boisaubin EV, Clark MA et al. Professional identity formation: Creating a longitudinal framework through TIME (transformation in medical education). Acad Med. 2015;90(6):761-7. doi: $10.1097 /$ ACM.0000000000000719

4. Wong A, Trollope-Kumar K. Reflections: An inquiry into medical students' professional identity formation. Med Educ. 2014;48(5):489-501. doi: 10.1111/medu.12382

5. Monrouxe LV. Identity, identification and medical education: Why should we care? Med Educ. 2010;44(1):40-49. doi: 10.1111/j.1365-2923.2009.03440.x

6. Pondé MP. Lazer e Saúde Mental. Salvador: Tempo Brasileiro;2007.

7. Bardin L. L'Analyse de Contenu. 7.ed. Paris: Presses Universitaires de France; 1993.

8. Burford B. Group processes in medical education: Learning from social identity theory. Med Educ. 2012;46(2):143-52. doi: 10.1111/j.1365-2923.2011.04099.x

9. Bandini J, Mitchell C, Epstein-Peterson ZD, Amobi A, Cahill J, Peteet J et al. Student and Faculty Reflections of the Hidden Curriculum. Am J Hosp Palliat Care. 2017;34(1):57-63. doi: 10.1177/1049909115616359

10. Martimianakis MA, Hafferty FW. Exploring the interstitial space between the ideal and the practised: humanism and the hidden curriculum of system reform. Med Educ. 2016;50(3):278-80. doi: $10.1111 /$ medu.12982

11. Wald HS. Professional identity (trans) formation in medical education: reflections, relantioship, resilience. Acad Med. 2015;90(6):701-6. doi: 10.1097/ACM.0000000000000731

12. Steinert Y, Cruess RL, Cruess SR, Boudreau JD, Fuks A. Faculty development as na instrument of change: a case study on teaching professionalism. Acad Med, 2007; 82(11):1057-64. doi: 10.1097/01.ACM.0000285346.87708.67 
13. Hopkins L, Saciragic L, Kim J, Posner G. The Hidden Curriculum: Exposing the Unintended Lessons of Medical Education. Cureus. 2016;8(10):e845. doi: 10.7759/cureus.845

14. Clandinin DJ, Cave MT. Creating pedagogical spaces for developing doctor professional identity. Med Educ, 2008;42(8):765-70. doi: 10.1111/j.1365-2923.2008.03098.x

15. Baird J, Bracken K, Grierson LEM. The relationship between perceived preceptor power use and student empowerment during clerkship rotations: a study of hidden curriculum. MedEduc, 2016;50(7):778-85. doi: 10.1111/medu.13065

16. Stern DT, Papadakis M. The developing physician - becoming a professional. N Engl J Med. 2006;355(17):1794-9. doi: 10.1056/ NEJMra054783

17. Balboni MJ, Bandini J, Mitchell C, Epstein-Peterson ZD, Amobi A, Cahill J et al. Religion, Spirituality, and the Hidden Curriculum: Medical Student and Faculty Reflections. J Pain Symptom Manage. 2015;50(4):507-15. doi: 10.1016/j.jpainsymman.2015.04.020
18. Hawick L, Cleland J, Kitto S. Sending messages: How faculty influence professionalism teaching and learning. Med Teach. 2017;39(9):987-994. doi: 10.1080/0142159X.2017.1337271

19. Wilkinson TJ. Stereotypes and the hidden curriculum of students. Med Educ. 2016;50(8):802-4. doi: 10.1111/medu.13008

20. Baird J, Bracken K, Grierson LEM. The relationship between perceived preceptor power use and student empowerment during clerkship rotations: a study of hidden curriculum. Med Educ. 2016;50(7):778-85. doi: 10.1111/medu.13065

21. Martimianakis MAT, Michalec B, Lam J, Cartmill C, Taylor JS, Hafferty FW. Humanism, the Hidden Curriculum, and Educational Reform: A Scoping Review and Thematic Analysis. Acad Med. 2015;90(11 Suppl):5-13. doi: 10.1097/ACM.0000000000000894 